\title{
Development prediction and the theoretical analysis in the ultra-high water cut stage for water-flooding sandstone reservoirs
}

\author{
Jianpeng $\mathrm{Xu}^{1} \cdot$ Chuanzhi Cui ${ }^{1} \cdot \mathrm{Zhiying} \mathrm{Ding}^{1} \cdot \mathrm{Peng}_{\mathrm{Xu}^{1}} \cdot \mathrm{Ronghua} \mathrm{Yao}^{1} \cdot$ \\ Peng Wang ${ }^{1}$
}

Received: 15 October 2015/Accepted: 27 March 2016/Published online: 21 April 2016

(c) The Author(s) 2016. This article is published with open access at Springerlink.com

\begin{abstract}
A new relationship between the ratio of water and oil relative permeability, and the water saturation is proposed based on the flow characteristic in porous media in the ultra-high water cut stage which results in a new water flooding characteristic curve with reservoir engineering method. Then a new algorithm to forecast development indexes is derived on the basis of the new relationship above, the new water flooding characteristic curve and the production decline law using Welge equation in which there are many parameters including time, water cut, the average water saturation and the oil recovery that can be used to evaluate the recoverable reserve. Through the examinations with the numerical simulation method and the on-site application, it shows that the new algorithm can give an accurate prediction with an relative error less than $6 \%$, which is instructive for the oil field development.
\end{abstract}

Keywords Ultra-high water cut - New flow characteristic relationship - Welge equation - Production decline law . Prediction

\section{Introduction}

Engineering methods and numerical simulation methods are widely used for the development prediction of water flooding reservoirs. The accurate application of the

Chuanzhi Cui

cuichuanzhi@126.com

Jianpeng Xu

xjpinupc@gmail.com

1 College of Petroleum Engineering, China University of Petroleum (East China), No. 66, Changjiang West Road, Qingdao, People's Republic of China numerical simulation is based on the detailed geological and production data which is time consuming. So reservoir engineering method is still widely used.

There have been plenty of papers on models for predicting development performance such as oil production and water cut (Li et al. 2011; Agarwal 1998; Haavardsson and Huseby 2007; Li and Horne 2005). However, less attention has been given to forecasting sweep efficiency and oil recovery. Actually it is critical for many aspects of reservoir engineering to accurately predict the developing performance. This includes reserves estimation, disposal of produced water, and reservoir surveillance and management. For oil reservoirs developed by water injection, especially those with low permeability or with a great density of fractures, prediction of water cut is still a big challenge. Currently, there are two main approaches (numerical simulation technique, and analytical or empirical method) to predict water cut. We focus on the latter approach in this study.

Water-flooding characteristic curves and production decline curves are used for performance analysis and prediction in water-flooding reservoirs (Chen 1993; Yu 1999; Gao and Xu 2007; Yang 2009; Dou et al. 2009; Arps 1945). The former can predict the accumulative water and oil production without the parameter of time. While the latter could illustrate the relationship between time and oil production which does not contain water cut and water production. To balance the cons and pros above, so many scholars tried combining water-flooding characteristic curves and production decline curves. Pei and Wang (1999) did some survey on the relationship between Arps decline curve and type I water-flooding characteristic curve. And then Hubbert model and water-flooding characteristic curve were combined to obtain the relationship between water cut and time (Chen and Zhao 2001). Wang (2001) developed the method with different indexes integrating 
type I type III water-flooding characteristic curves and a production prediction model. Later, a new model was built to predict different development indexes based on $\Gamma$ model and type I water-flooding characteristic curve (Zhao and Chen 2004; Xu and Chen 2005). Recently different waterflooding characteristic curves and Weibull model were combined and the optimal one was screened out (Liu et al. 2013). While oil saturation could decline further in the ultra-high water cut stage, the declining oil saturation results in the relative permeability to water and oil changing dramatically which brings errors when using general forecasting algorithm applicable to the medium and high water cut stage (less than $90 \%$ ). For the particularity of water and oil permeability caused by many factors in the ultra-high water cut stage several scholars built different formulas to describe it such as quadratic and exponent equations. In this paper a new model is developed on the basis of the new water-flooding curve and Arps decline curve, and then the evaluation is conducted using reservoir numerical simulation method and the on-site data.

\section{Establishment of the prediction model}

\section{Production decline law}

There are many kinds of models for production prediction such as decline curve method and growth curve method. Growth curve method is usually conservative in the ultrahigh water cut stage while Arps decline curve is applicable for constant pressure condition and constant liquid condition in ultra-high water cut stage (Zhang 2013).

Arps decline equation was developed using statistical analysis by Arps and the theoretical derivation was provided by Ji (1995).

The oil production rate in the decline period is as follows:

$q_{d}=\frac{q_{i}}{\left(1+b D_{i} t\right)^{1 / b}}$

Equation (1) gives the oil production rate during the decline period including decline factor, $D_{i}$ and decline exponent, $b$.

The total cumulative oil production can be written as:

$N_{\mathrm{p}}=N_{\mathrm{p} 0}+N_{\mathrm{pd}}$

Equation (2) describes the cumulative oil production containing cumulative oil production during the decline period, $N_{\mathrm{pd}}$ and the cumulative oil production containing cumulative oil production before the decline period, $N_{\mathrm{p} 0}$.

\section{Water flooding prediction model}

There are dozens of water-flooding characteristic curves including four typical curves whose theoretical basis is the linear relationship between the ratio of water and oil relative permeability and water saturation in the semi-logarithmic coordinate. However, the straight line will bend down in the ultra-high water cut stage where water and oil permeability have dramatic changes. So we characterize the changes with Eq. (4) as follows:

$\lg \mathrm{WOR}=A+B N_{p}+C N_{p}^{2}+\frac{D}{1-S_{\mathrm{or}}-S_{\mathrm{wc}}-E N_{p}}$

where WOR is water/oil mass ratio, $S_{\text {or }}$ is the irreducible oil saturation, $S_{\mathrm{wc}}$ is the connate water saturation, $S_{\mathrm{we}}$ is the outlet water saturation and the others are different coefficients. Equation (4) is a new formula that can elucidate the new relationship between $k_{\text {ro }}$ and $k_{\text {rw }}$ in the ultra-high water cut stage.

$\ln \frac{k_{\mathrm{ro}}}{k_{\mathrm{rw}}}=a+b S_{\mathrm{we}}+c S_{\mathrm{we}}^{2}+\frac{d}{1-S_{\mathrm{or}}-S_{\mathrm{we}}}$

The water fractional flow represented by $f_{\mathrm{w}}$ can be derived using Darcy law as follows:

$f_{\mathrm{w}}=\frac{Q_{\mathrm{w}}}{Q_{\mathrm{o}}+Q_{\mathrm{w}}}=\frac{1}{1+\frac{1}{\mathrm{WOR}}}=\frac{1}{1+\frac{k_{\mathrm{ro}}}{k_{\mathrm{rw}}} \mu_{\mathrm{o}}}$

where $Q_{\mathrm{w}}$ is the water production rate, $\mathrm{m}^{3} / \mathrm{d} ; Q_{\mathrm{o}}$ is the oil production rate, $\mathrm{m}^{3} / \mathrm{d} ; k_{\mathrm{ro}}$ is the oil relative permeability, $k_{\mathrm{rw}}$ is the water relative permeability.

Substituting Eqs. (3) and (4) into Eq. (5) gives

$f_{w}=1-\frac{1}{1+10^{A+B N_{\mathrm{p}}+C N_{p}^{2}+\frac{D}{1-S_{\mathrm{or}}-S_{\mathrm{wc}-E N_{\mathrm{p}}}}}}$

and

$f_{\mathrm{w}}=\frac{1}{1+\frac{\mu_{\mathrm{w}}}{\mu_{\mathrm{o}}} \cdot \exp \left(a+b S_{\mathrm{we}}+c S_{\mathrm{we}}^{2}+\frac{d}{1-S_{\mathrm{or}}-S_{\mathrm{we}}}\right)}$

Rewriting Eq. (7) we can get

$$
\begin{aligned}
& {\left[\ln \frac{\mu_{\mathrm{o}}}{\mu_{\mathrm{w}}}\left(\frac{1}{f_{\mathrm{w}}}-1\right)-a+b\left(1-S_{\mathrm{or}}\right)\right] S_{\mathrm{we}}} \\
& \quad+\left[\left(1-S_{\mathrm{or}}\right) c-b\right] S_{\mathrm{we}}^{2}-c S_{\mathrm{we}}^{3} \\
& \quad=\left(1-S_{\mathrm{or}}\right)\left[\ln \frac{\mu_{\mathrm{o}}}{\mu_{\mathrm{w}}}\left(\frac{1}{f_{\mathrm{w}}}-1\right)-a\right]-d
\end{aligned}
$$

Equation (8) is a simple cubic equation which could be solved easily. And the outlet water saturation can be represented by water cut using a function as follows:

$S_{\mathrm{we}}=G\left(f_{\mathrm{w}}\right)$

Differentiate Eq. (8) and then we get 
$\frac{d f_{\mathrm{w}}}{d S_{\mathrm{we}}}=\left(b+2 c S_{\mathrm{we}}+\frac{d}{1-S_{\mathrm{or}}-S_{\mathrm{wc}}}\right)\left(f_{\mathrm{w}}^{2}-f_{\mathrm{w}}\right)$

The relationship between the average water saturation and the water saturation in the outlet was given by Welge (1952), as follows:

$\overline{S_{\mathrm{w}}}=\frac{\int_{x_{1}}^{x_{2}} S_{\mathrm{w}} d x}{x_{2}-x_{1}}=S_{\mathrm{we}}+\frac{1-f_{\mathrm{w}}}{f_{\mathrm{w}}^{\prime}\left(S_{\mathrm{we}}\right)}$

Rewriting Eqs. (11) and (10), the result is as follows

$$
\begin{aligned}
\overline{S_{\mathrm{w}}}= & S_{\mathrm{we}}+\left(b+2 c S_{\mathrm{we}}+\frac{d}{1-S_{\mathrm{or}}-S_{\mathrm{wc}}}\right) \\
& \times\left(f_{\mathrm{w}}^{2}-f_{\mathrm{w}}\right)\left(1-f_{\mathrm{w}}\right)
\end{aligned}
$$

The oil recovery is defined as the ratio of cumulative oil production to initial oil in place. So it can be indicated with water saturation based on material balance equation as shown in Eq. (13) where $R$ is the oil recovery.

$$
\begin{aligned}
R & =\frac{\overline{S_{\mathrm{w}}}-S_{\mathrm{wi}}}{1-S_{\mathrm{wi}}} \\
& =\frac{\left(b+2 c S_{\mathrm{we}}+\frac{d}{1-S_{\mathrm{or}}-S_{\mathrm{wc}}}\right)\left(f_{\mathrm{w}}^{2}-f_{\mathrm{w}}\right)\left(1-f_{\mathrm{w}}\right)}{1-S_{\mathrm{wi}}}+\frac{S_{\mathrm{we}}-S_{\mathrm{wi}}}{1-S_{\mathrm{wi}}}
\end{aligned}
$$

The oil recovery is also the product of sweep efficiency and displacement efficiency, and therefore sweep efficiency is available given oil recovery and displacement efficiency.

$R=E_{D} \times E_{V}$

$E_{\mathrm{D}}=\frac{S_{\mathrm{oi}}-S_{\mathrm{or}}}{S_{\mathrm{oi}}}$

In Eq. (14), $E_{D}$ is the displacement efficiency and $E_{V}$ is sweep efficiency.

Integrating Eq. (13) to Eq. (15), sweep efficiency can be obtained as follows:

$$
\begin{aligned}
E_{V}= & \frac{\left(b+2 c G\left(f_{\mathrm{w}}\right)+\frac{d}{1-S_{\mathrm{or}}-S_{\mathrm{wc}}}\right)\left(f_{\mathrm{w}}^{2}-f_{\mathrm{w}}\right)\left(1-f_{\mathrm{w}}\right)}{\left(1-S_{\mathrm{wi}}\right)\left(S_{\mathrm{oi}}-S_{\mathrm{or}}\right)} \\
& +\frac{G\left(f_{\mathrm{w}}\right)-S_{\mathrm{wi}}}{\left(1-S_{\mathrm{wi}}\right)\left(S_{\mathrm{oi}}-S_{\mathrm{or}}\right)} \times S_{\mathrm{oi}}
\end{aligned}
$$

Production decline law gives the relationship between time $(t)$ and cumulative oil production $\left(N_{\mathrm{p}}\right)$, and we have derived the equation with regard to cumulative oil production $\left(N_{\mathrm{p}}\right)$, water cut $\left(f_{\mathrm{w}}\right)$, oil recovery $(R)$ and sweep efficiency. As shown in Fig. 1, production decline and water-flooding curves could be combined to forecast many development indexes and estimate the oil initially in place(Reserve) using cumulative oil production and oil recovery calculated above.

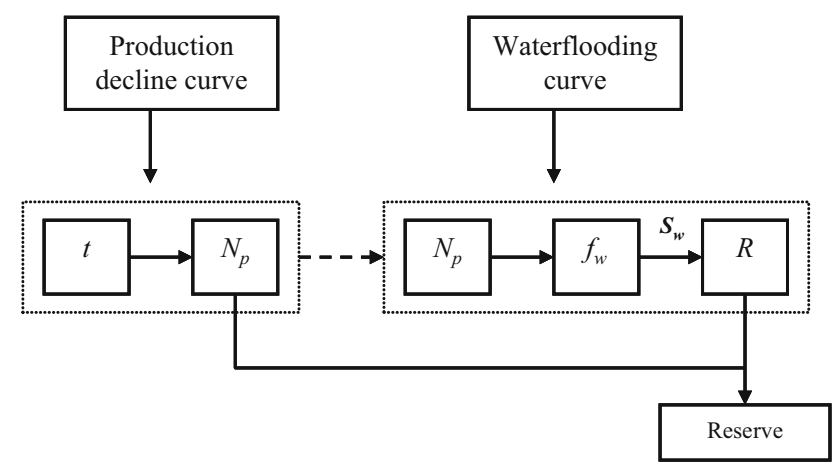

Fig. 1 The flow chart

\section{Theoretical basis}

There are several characteristics in the ultra-high water cut stage for water-flooding reservoirs different from that in medium water cut stage such as the condition of remaining oil and the reservoir quality. During water injection the remaining oil in the formation gets less and less and water saturation gradually increases so that the remaining oil becomes discrete in the ultra-high water cut stage. Displacement experiments indicates that water injection can cause changes in reservoir quality and the reason is that fine particle and sialite remove from the rock skeleton and get out of production wells with the liquid in the unconsolidated reservoir that is lack of clay minerals. These changes result in an increase in porosity and permeability. Moreover the fluid may react with the constituents of the formation surface which has influence on the wettability of the formation and the degree of the change has correlation with water cut (Wang et al. 2013) as shown in Table 1.

The high-powered relative permeability curve is also of importance in the research on the flow characteristics in porous media in the ultra-high water cut stage.

Compared with the general relative permeability curve the high-powered one can accurately reflect the characteristic that occurs in the ultra-high water cut stage during which time the isoperm point moves to the right and the residual oil saturation has a drop as shown in Fig. 2 which is the reason why water and oil relative permeability change dramatically. And the linear relationship between $\ln \left(k_{\mathrm{ro}} / k_{\mathrm{rw}}\right)$ and $S_{\mathrm{w}}$ is not the truth (Yang et al. 2013) which is common in medium and high water cut stage. So models based on that linear relationship are rarely used.

Some investigators thought that the nonlinear flow in the strongly water flushed zone of the porous media was the reason why strange flow characteristics occurred in the ultra-high water cut stage (Zhang 2009). And systematical analysis was given in regard to different conditions and 
Table 1 Contact angle at different water cut

\begin{tabular}{lccll}
\hline Well name & \multicolumn{4}{l}{ Contact angle $/\left(^{\circ}\right)$} \\
\cline { 2 - 5 } & $f_{\mathrm{w}}=0$ & $f_{\mathrm{w}}=60 \%$ & $f_{\mathrm{w}}=80 \%$ & $f_{\mathrm{w}}=90 \%$ \\
\hline G206-4 & 42.3 & 105.4 & 83.7 & 75.9 \\
G190X1 & 106.9 & 93.3 & 47.9 & 33.6 \\
LN2-6 & 29.3 & 65.1 & 44.3 & 33.4 \\
\hline
\end{tabular}

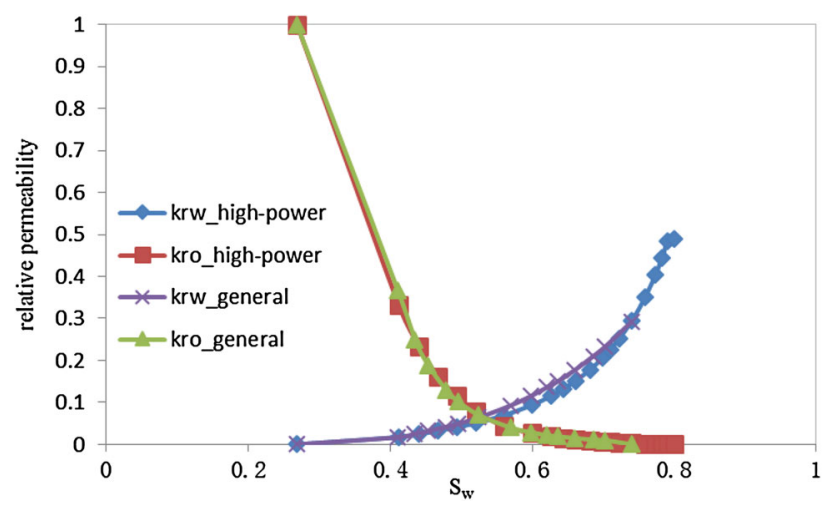

Fig. 2 Comparison of different relative permeability curves

factors. Some scholars presented a piecewise equation to describe the flow characteristics in the ultra-high water cut stage while the turning point connecting the two different formula was hard to determine. In addition, a nonlinear formula shown in Eq. (17) was presented to interpret the data from high-powered relative permeability curves which gives an approach to confirm the residual oil saturation in the water flushed zone (Yu 2014).

$\ln \frac{k_{\mathrm{ro}}}{k_{\mathrm{rw}}}=R \ln \left(m-n S_{\mathrm{w}}\right)$

In this paper a new relationship indicated as Eq. (4) between the ratio of water and oil relative permeability, and water saturation is launched which is reasonable. There are quadratic term and the reciprocal of the term, $\left(1-S_{\text {or }}-S_{\text {we }}\right)$ in the new formula compared with the general one as shown in Eq. (18).

$\ln \frac{k_{\mathrm{ro}}}{k_{\mathrm{rw}}}=m+n S_{\mathrm{we}}$

The quadratic term can make the formula adapt to the real condition better. The value of $\ln \left(k_{\mathrm{ro}} / k_{\mathrm{rw}}\right)$ approaches to infinite when water saturation is close to $\left(1-S_{\text {or }}\right)$ at which the reciprocal of $\left(1-S_{\text {or }}-S_{\text {we }}\right)$ is used. So the new equation has physical basis and in addition, we get nice results when fitting several relative permeability curves shown in Fig. 3 where the correlation coefficient is more than 0.99 .

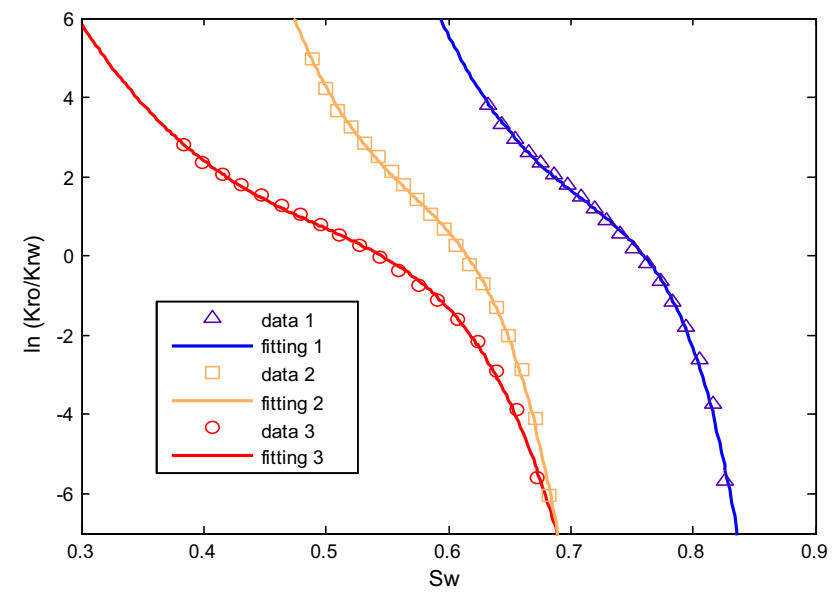

Fig. 3 Fitting curves of the relative permeability with the new relationship

\section{Parameters solution}

When a reservoir is in the ultra-high water cut stage, the production rate decline markedly and meanwhile, trialand-error method is usually used to determine decline factor (Jiang et al. 2006). Then the relationship between cumulative oil production and time can be obtained by substituting decline factor to Eq. (2). For the fitness of relative permeability curves and water-flooding curves, the nonlinear terms should be transformed into linear terms firstly which is the precondition to solve the equation using multiple linear regression (Chang et al. 2009).

\section{Validation and application}

\section{Reservoir simulation}

A geological model is built in the light of a block in Shengli oil field and the basic parameters are in Table 2. And then we conduct history match and development prediction during reservoir simulation.

Production data, like water cut, cumulative oil, oil recovery, is collected after finishing numerical simulation with the model above.

To predict the performance in the ultra-high water cut stage the data before water cut exceeds $85 \%$ is used to match the parameters. Through analyzing the data, the initial decline time and producing rate are confirmed and meantime, we obtain $Q_{0}=2884.3 \mathrm{~m}^{3} / \mathrm{mon}$ and $N_{p 0}=11,773.4 \mathrm{~m}^{3}$. The value of decline exponent and initial decline factor is 0.54 and $0.1087 \mathrm{mon}^{-1}$ respectively, and the relationship between cumulative oil production and time can be written correspondingly as: 
Table 2 Basic parameters of the reservoir property

\begin{tabular}{llll}
\hline Parameter & Value & Parameter & Value \\
\hline $\begin{array}{l}\text { Density of water } \\
\left(\mathrm{g} / \mathrm{cm}^{3}\right)\end{array}$ & 1 & $\begin{array}{c}\text { Density of } \\
\text { oil }\left(\mathrm{g} / \mathrm{cm}^{3}\right)\end{array}$ & 0.867 \\
$B_{\mathrm{o}}$ & 1.14 & $B_{\mathrm{w}}$ & 1.02 \\
$S_{\mathrm{w}}$ & 0.3 & $S_{\mathrm{or}}$ & 0.18 \\
$\begin{array}{l}\text { Viscosity of water } \\
\quad(\mathrm{mPa} \mathrm{s})\end{array}$ & 0.4 & $\begin{array}{l}\text { Viscosity of water } \\
(\mathrm{mPa} \mathrm{s})\end{array}$ & 8 \\
\hline
\end{tabular}

$$
\begin{aligned}
N_{\mathrm{p}}= & \frac{Q_{0}}{0.1087(0.54-1)}\left[\left(1+0.0587 t_{d}\right)^{\frac{0.54-1}{0.54}}-1\right] \\
& +11773.397
\end{aligned}
$$

The equations of water-flooding curves and relative permeability curves are obtained with multiple correlation method which can be used to forecast development performance, for example, water cut shown as Fig. 4, in the ultra-high water cut stage combined with the production decline curve shown as Eq. (19).

The relative error of water cut and oil recovery is defined in Eqs. (20) and (21)

$\operatorname{error}_{f_{\mathrm{w}}}=\frac{f_{\mathrm{w}}^{\mathrm{cal}}-f_{\mathrm{w}}^{*}}{f_{\mathrm{w}}^{*}}$

$\operatorname{error}_{R}=\frac{R_{\mathrm{cal}}-R^{*}}{R^{*}}$

where $f_{\mathrm{w}}^{\mathrm{cal}}$ is the predicting value of water cut, $f_{\mathrm{w}}^{*}$ is the actual water cut value, $R_{\mathrm{cal}}$ is the predicting value of oil recovery, and $R^{*}$ is the actual value oil recovery.

Figure 5 shows that the ability to predict water cut in the ultra-high water cut stage of differnet models varies and the result from the linear model is conservative. The relative error from the new model is decreasing gradually with time which is less than $5 \%$ in the ultra-high water cut stage in Fig.6.

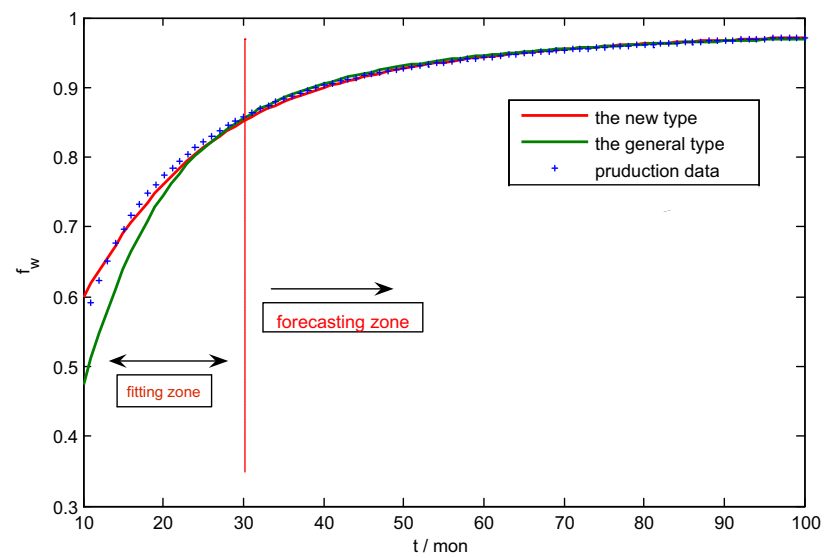

Fig. 4 The forecasting chart for water cut comparison of different relative permeability curves

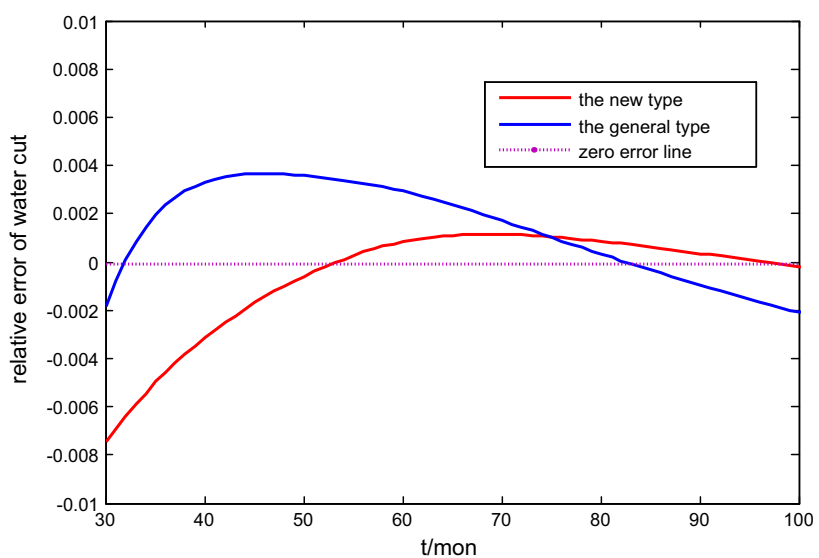

Fig. 5 The relative error diagram of water cut

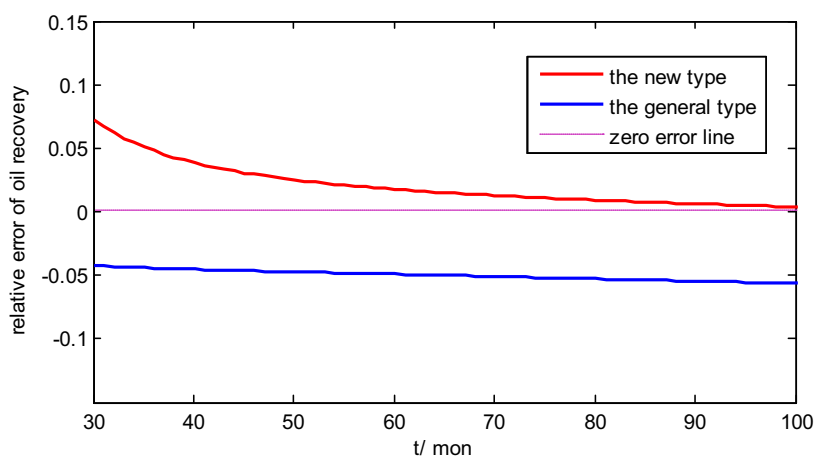

Fig. 6 The relative error diagram of recovery

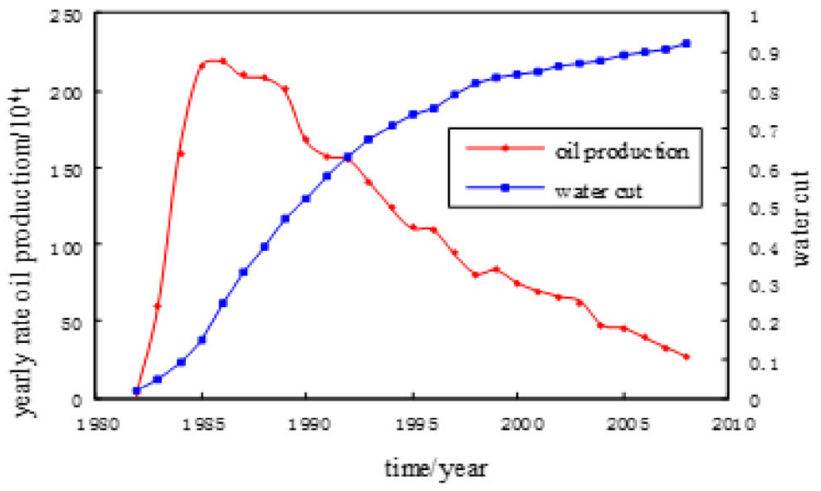

Fig. 7 The on-site production data

The prediction trend of oil recovery is similar to that of water cut where the new model is more accurate with a relative error less than $2 \%$ after 60 months.

The solution from the new model can forecast the development performance accurately which indicates that 


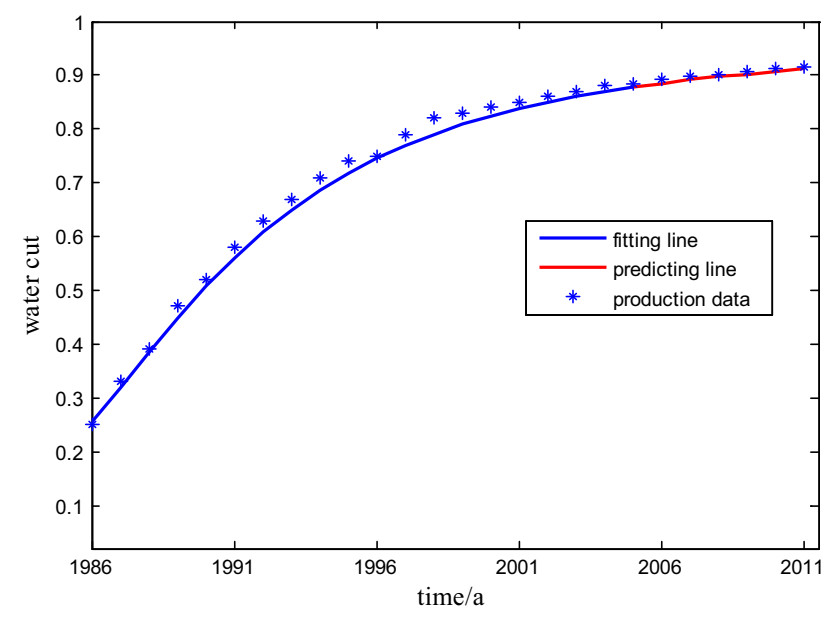

Fig. 8 The forecasting diagram of water cut

the new equation presented adapts to the flow characteristics in porous media in the ultra-high water cut stage.

\section{On-site application}

The data from the paper ( $\mathrm{Bi} 2007$ ) is used to verify the new model which is indicated in Fig. 7. The oil-bearing area equals $25.31 \mathrm{~km}^{2}$ and the reserve is $7076.41 \times 10^{4} \mathrm{t}$. The initial decline time, decline exponent and decline factor can be obtained through trial-and-error. The prediction of water cut is shown in Fig. 8 in which the relative error is less than $4 \%$.

\section{Discussion}

It is inevitable that water cut is increasing gradually for water-flooding reservoirs so it makes sense to investigate the flow mechanism in porous media for the purpose of keeping water cut and oil production in ultra-high water cut stage. The specific performance of different flow mechanism in porous medium during ultra-high water cut period lies in the condition of the remaining oil and formation property. Some researchers hold the view that the property of the subsurface fluid may changes for water injection especially in the strongly water flushed zone. Then many experiments about subsurface fluid property at different water cut stages are conducted and relevant reservoir numerical simulation is also developed. Most of matured fields in east of China such as Daqing and Shengli oil fields are continental sedimentation that easily go into the ultra-high water cut stage after dozens of years.

For the flow mechanism in heavily water flushed porous media there are many core flooding experiments, while the study only lies in the data fitting with the inner mechanism less involved.

In the past the core flooding experiment was completed when the volume of injected water reached 30 pore volumes of the core according to the standard, SY/T53452007. In fact there is considerable amount of incremental oil produced if we keep on water-injecting, which is the origin of the high-powered relative permeability curve. In the ultra-high water cut stage, factors affecting displacement efficiency were investigated through abnormal coreflooding experiments in which the residual oil saturation decreases as water cut rises which is inversely proportional to capillary number, as follows:

$N_{\mathrm{c}}=\frac{v \mu_{\mathrm{w}}}{\sigma}$

where $N_{\mathrm{c}}$ is capillary number, $v$ is velocity and $\sigma$ is interfacial tension. So we can suppose that $\sigma$ in Eq. (22) may get affected in the ultra-high water cut stage.

Ji et al. (2011) analyzed the factors affecting the capillary number during ultra-high water cut period and indicated the research direction of displacement efficiency. Liu et al. (2011), Wang et al.(2013) and Song et al. (2013) investigated the flow characteristic in porous media in the ultra-high water stage and built different relative permeability formulas and waterflood curves. In this paper a new model based on the new relationship of water and oil relative permeability is raised which is physically meaningful and owns a quick efficient and reliable quality to deal with the on-site data.

But there are still something insufficient for the flow mechanism in the porous media in the ultra-high water cut stage. The existing models are developed based on Darcy Law which does not adapt to the ultra-high water cut condition in which the phase of oil is discrete. The changes of formation property are not taken into account in the models above so the fluid-structure coupling in the ultrahigh water cut stage should be the focus in the future.

\section{Conclusion}

The special flow characteristic in porous media in the ultrahigh water stage is well depicted by the nonlinear relationship between $\ln \left(k_{\mathrm{ro}} / k_{\mathrm{rw}}\right)$ and $S_{\mathrm{we}}$ the reason of which includes wettability change, fine particles migration and non-Darcy flow in the zone of high permeability.

A new approach of predicting water cut, average water saturation, oil recovery and sweep efficiency is presented using the production decline curves and the new relative relationship $\ln \left(k_{\mathrm{ro}} / k_{\mathrm{rw}}\right)$ and $S_{\mathrm{we}}$ which can also estimate the reserve given cumulative oil production and oil recovery. Reservoir simulation and field application show that the 
new model conforms to the ultra-high water cut stage very well whose forecasting error gets smaller and smaller with time.

Acknowledgments The authors wish to thank the China National Science and Technology Major Projects (Grant No: 2016ZX05011) and Changjiang Scholars and Innovative Research Team in University (IRT1294) for the financial support during research.

Open Access This article is distributed under the terms of the Creative Commons Attribution 4.0 International License (http:// creativecommons.org/licenses/by/4.0/), which permits unrestricted use, distribution, and reproduction in any medium, provided you give appropriate credit to the original author(s) and the source, provide a link to the Creative Commons license, and indicate if changes were made.

\section{References}

Agarwal RG (1998) Analyzing well production data using combined type curve and decline curve analysis concepts. SPE Reserv Eval Eng 2(5):478-486

Arps JJ (1945) Analysis of decline curves. Trans AIME 160:228-247

Bi YB (2007) Predicting development index of daqing Mid-Sa oilfield at later high water stage. Dissertation, Daqing Petroleum Institute

Chang ZG, Wang QH, and Du CF (2009) Application of statistical methods. Beijing

Chen YQ (1993) Derivation of a new type of water displacement curve and it's application. Acta Petrolei Sin 14(2):65-73

Chen YQ, Zhao QF (2001) A combined method of Hubbert model and water drive curve. China Offshore Oil Gas 15(3):194-199

Dou $\mathrm{H}$ et al (2009) Analysis and comparison of decline models: a field case study for the intercampo oil field, venezuela. SPEREE 12(1):68-78

Gao WJ, Xu J (2007) Theoretical study on common water-drive characteristic curves. Acta Petrolei Sin 28(3):89-92

Haavardsson NF, Huseby AB (2007) Multisegment production profile models - a tool for enhanced total value chain analysis. J Petrol Sci Eng 58(1):325-338

Ji BY (1995) The fundamentals of seepage flow theory used on the production decline equations. Acta Petrolei Sin 16(3):86-91
Ji SH, Tian CB, Chengfang (2011) New understanding on water-oil displacement efficiency in a high water-cut stage. Pet Explor Dev 38(3):338-345

Jiang HQ, Yao J, and Jiang RZ (2006) Principle and method of reservoir engineering, Dongying

Li KW, Horne RN (2005) An analytical model for production declinecurve analysis in naturally fractured reservoirs. SPE Reserv Eval Eng 8(3):197-204

Li KW, Ren XH, Li Li, Fan XD (2011) A new model for predicting water cut in oil reservoirs. SPE 143481

Liu SH, Gu J, Yang R (2011) Peculiar water-flooding law during high water-cut stage in oilfield. J Hydrodyn 26(6):660-666

Liu F, Du Z, Chen X (2013) Combining water flooding type-curves and Weibull prediction model for reservoir production performance analysis. J Petrol Sci Eng 112(1):220-226

Pei LJ, Wang ZL (1999) Correlativity of Arps production decline curve vs. Type A water drive curve and their parameter calculation. Pet Explor Dev 26(3):62-65

Song Z, Li Z, Lai F (2013) Derivation of water flooding characteristic curve for high water-cut oilfields. Pet Explor Dev 40(2):201-208

Wang JK (2001) A Joint application of displacement curve and production prediction model. XIPG 22(5):426-428

Wang RR, Hou J, Li ZQ (2013) A New water displacement curve for the high water-cut stage. Pet Sci Technol 31(13):1327-1334

Welge HJ (1952) A simplified method for computing oil recovery by gas or water drive. AIME 195:91-98

Xu XF, Chen XG (2005) Application of the combined solution model based on $\Gamma$ model and water Drive curves. Comput Simul 22(1):227-229

Yang Z (2009) A new diagnostic analysis method for waterflood performance. SPEREE 12(2):341-351

Yang C, Li YL, Xu BX (2013) New nonlinear correction method of oil-water relative permeability curves and their application. Oil Gas Geol 34(3):394-399

Yu QT (1999) Characteristics of oil-water seepage flow several important water drive curves. Acta Petrolei Sin 20(1):56-60

Yu CL (2014) A new prediction method of relative permeability to reflect water-flooding limitation. Special Oil Gas Reserv 21(2):123-126

Zhang XS (2009) Generating condition of non-linear flow in waterflooding reservoir at ultra-high water-cut stage. J China Univ Pet (Ed Nat Sci) 33(2):90-93

Zhang JQ (2013) Production forecasting models of waterflood oilfield, Beijing

Zhao F, Chen XG (2004) A simultaneous solving process for $\Gamma$ model and water drive curve. Special Oil Gas Reserv 11(1):44-46 